\title{
Églises en éclats : le temps des débats et des partenariats \\ Pour une nouvelle gouvernance \\ du patrimoine religieux en milieu rural
}

\author{
Claude Pigeon, Diocèse de Rimouski \\ Majella Simard, Université du Québec à Rimouski
}

\section{Introduction}

$\mathcal{L}$ a société québécoise est consciente qu'elle doit rapidement prendre position face à l'avenir de son patrimoine religieux bâti. Laissées à elles-mêmes, les communautés paroissiales ne peuvent plus soutenir la mission première de l'Église, à savoir l'évangélisation, tout en remplissant adéquatement son mandat de conservation de plusieurs édifices, églises ou presbytères, héritage d'une situation de chrétienté qui englobait la quasi-totalité de la société. La nécessité de convertir, de louer, de vendre ou même de démolir des églises, que l'on pensait le fait des grands centres, rejoint rapidement les petites paroisses du monde rural. Les responsables de l'Église et les participants réguliers à la vie paroissiale constituent une minorité du quartier ou du village. Peuvent-ils prendre seuls des décisions qui concernent un héritage collectif? Telle est la question qui alimentera notre réflexion dans le cadre de cet article. Le temps est aux débats ouverts entre citoyennes et citoyens, aux assemblées de paroisses ou de quartiers, et aux recherches de partenariats.

L'objectif de cette contribution est de mieux cerner les principaux enjeux liés à la préservation du patrimoine religieux en milieu rural. Notre démarche comporte cinq volets. Dans un premier temps, nous évoquerons la prise de conscience collective qui se manifeste présentement à travers tout le Québec en ce qui a trait à la sauvegarde de ce patrimoine. En milieu rural, celui-ci exerce une fonction symbolique indéniable en termes d'appartenance territoriale et d'identité collective. La deuxième partie jettera un regard plus sociologique à cet égard. Dans les troisième et quatrième parties, nous examinerons quelques initiatives mises en œuvre par les populations locales dans le but d'apporter un nouveau souffle aux infrastructures et aux services religieux. Enfin, nous terminerons en énonçant quelques pistes ou avenues de solutions à privilégier.

\section{Une rapide prise de conscience collective}

Au cours des derniers mois, la sensibilité des Québécois a été rapidement éveillée par une importante couverture médiatique de la situation actuelle de leur patrimoine religieux bâti. Plusieurs articles, lettres ouvertes ou reportages télévisés ont fait connaître les inquiétudes de la population de certains quartiers ou villages face à l'inévitable fermeture ou changement de vocation d'églises paroissiales ou d'institutions religieuses comme le clô̂tre du Carmel, à Montréal. Les universitaires et les décideurs ne sont pas en reste dans leur désir d'identifier les enjeux en cause et de rechercher des orientations ou des solutions à proposer. Par exemple, du 19 au 22 octobre 2005, chercheurs et acteurs locaux ont repris les expériences locales et l'expertise internationale pour les mettre en commun dans le cadre du colloque «Quel avenir pour quelles églises? » organisé par l'École des sciences de la gestion de l'UQAM, la Fondation du patrimoine religieux, le Conseil du patrimoine et l'Université Concordia. De son côté, la Commission de la culture du gouvernement du Québec, présidée par le député de Shefford, Bernard Brodeur, parcoure le territoire québécois et se penche sur la préservation du patrimoine religieux québécois. Les dépositaires actuels, les Églises et groupes religieux, ne pouvant plus remplir leur cahier de charges au titre de la préservation 
des édifices, la question se pose d'une sélection et des moyens mis en œuvre pour conserver des infrastructures.

Au Québec, en milieu rural en particulier, la fragilisation du patrimoine religieux bâti repose sur une convergence de plusieurs causes déjà bien connues telles que l'exode et le vieillissement de la population, le désintérêt des jeunes et des adultes en ce qui regarde la vie paroissiale, ou la diminution importante des revenus des paroisses. Plus récemment, se sont ajoutés d'autres facteurs qui rendent incontournable une prise de décision rapide et accentuent, en ce domaine, le sentiment d'urgence, parfois même de crise. Mentionnons d'abord l'explosion des coûts d'énergie qu'on peut attribuer à l'augmentation de $100 \%$ du prix de l'huile à chauffage depuis 2003 et à l'abolition, en avril 2006, du tarif biénergie pour les églises, qui devrait entraîner, au minimum, une augmentation de $200 \%$ des coûts. Notons également, dans plusieurs cas, l'entretien des bâtiments qui a été négligé au cours des dernières années, faute de ressources humaines et matérielles. Ce fait est actuellement mis en lumière par le resserrement des normes de sécurité et des conditions plus strictes pour le renouvellement des polices d'assurance. Soulignons, enfin, le besoin plus grand de personnel formé et rémunéré pour coordonner ou assurer la catéchèse des jeunes catholiques d'âge scolaire dont la responsabilité, avec l'adoption de la loi 118, a été rendue aux paroisses et aux familles.

\section{Avec le cimetière, et à un degré moindre le presbytère, l'église joue un rôle structurant dans l'organisation de l'espace en milieu rural.}

\section{Un patrimoine religieux aux incidences sociales}

L'Église catholique au Québec a été à l'origine de la constitution d'un fabuleux patrimoine architectural religieux, avec ses diverses institutions, églises et presbytères répartis sur tout le territoire. En milieu rural, le clocher du village continue de jouer un rôle catalyseur. Sans faire de mauvais jeu de mots, il continue de faire résonner un héritage à la fois chrétien et citoyen. Il signale bien sûr la présence chrétienne, élément incontournable de notre histoire et réalité tou- jours importante pour une tranche non négligeable de la population. Mais plus encore peut-être, il fait écho à la force de caractère, aux valeurs et aux traditions des femmes et des hommes qui ont bâti une région, à la détermination et à la solidarité d'une collectivité qui, contre vents et marées, s'est mobilisée et continue de s'engager activement pour maintenir son identité particulière et ses moyens de subsistance. L'église du village québécois, qui possède souvent des qualités architecturales indéniables, porte encore bien haut, pour sa population, les idéaux d'un terroir faits d'une histoire, d'une culture, de traditions familiales et de solidarités humaines extraordinaires. Avec le cimetière, et à un degré moindre le presbytère, l'église joue un rôle structurant dans l'organisation de l'espace en milieu rural ${ }^{1}$. Ils constituent un symbole identitaire important. Témoin des luttes passées, l'église paroissiale est l'expression physique des racines de toute une communauté.

Ce n'est pas parce qu'une institution n'est plus en mesure d'assurer à elle seule le maintien, la conservation et la mise en valeur d'un ou de plusieurs bâtiments que ces derniers perdent leur valeur ou leur force symbolique. Bien des citoyens, indépendamment de leur appartenance ou référentiel religieux, gardent en mémoire des récits heureux ou tristes de leur histoire familiale liés à la construction et à l'entretien ou à la vie sociale et religieuse qui s'est déroulée à l'ombre du clocher du village. Indépendamment de l'adhésion aux discours ou aux pratiques religieuses, un lien sociologique et affectif durable est établi en vue de la reconnaissance par le plus grand nombre du patrimoine religieux comme héritage collectif. À ce titre, les décisions concernant l'avenir interpellent toutes les tranches de la population et doivent faire l'objet de débats publics locaux et nationaux.

La Commission sur la culture du gouvernement du Québec est un pas dans la bonne direction afin d'éveiller la conscience collective sur les enjeux et les conséquences irréversibles des décisions qui se prennent actuellement. Quant aux responsables de l'Eglise catholique, au titre de dépositaires actuels de ce patrimoine, ils doivent développer une attitude facilitant les débats ouverts au sein de l'ensemble de la population. Si l'on admet le principe de l'héritage collectif lorsqu'il s'agit de solliciter les offrandes de la population, il faut aller jusqu'au bout et reconnaître son droit de parole à l'heure des choix. 
Il peut sembler légitime de questionner les choix d'une époque où la population n'était pas toujours partie prenante des processus décisionnels. Mais les décisions concernant l'avenir de nos églises se prendront-elles comme ont été prises celles concernant leur construction, sans véritable consultation populaire ? On ne corrigera pas certaines erreurs du passé en les reproduisant.

\section{Un processus déjà en cours}

Depuis les années 1990, on a assisté à la vente de nombreux presbytères et couvents. Leur changement de vocation a contribué à conférer une ou des fonctions nouvelles, le plus souvent à caractère touristique, ce qui, par ricochet, a entraîné une diversification de l'offre de services locaux. Les microprojets de développement local, en cette matière, sont relativement nombreux. Pour l'essentiel, ils prennent la forme de gîtes du passant, de musées, de galeries d'art, de cafés, de petits restaurants, de logements sociaux ou de résidences pour personnes âgées. Ils concernent autant les milieux ruraux dynamiques que ceux présentant des signes de dévitalisation. Citons quelques exemples fournis par la région du Bas-St-Laurent. D'abord, la petite municipalité de Sainte-Florence, dans la Vallée de la Matapédia, où le presbytère a été transformé en gîte du passant, contribuant ainsi à consolider le volet touristique de cette localité fréquentée surtout par des amateurs de chasse et de pêche. Ensuite, dans la MRC des Basques, le presbytère de SaintÉloi a été transformé en restaurant gastronomique qui met en valeur l'agneau régional. Puis, à Sainte-Flavie, aux portes de la Gaspésie, le presbytère municipalisé fournit un toit à la bibliothèque locale et à une galerie d'art ouverte aux artistes du milieu. Mentionnons, enfin, le presbytère de Saint-Mathieu-de-Rioux qui a été acquis par la Société d'exploitation des ressources (SER) qui l'a restauré et y a installé ses bureaux. Au plan des institutions, la conversion du couvent de Saint-Casimir, dans la MRC de Portneuf, apporte l'exemple de la création d'une auberge multifonctionnelle. Mais la vente des édifices religieux n'est pas toujours possible. Ceux-ci sont alors laissés à leur propre sort ou bien continuent de monopoliser la plus grande partie des revenus de la fabrique, contribuant ainsi à la réduction des ressources affectées à l'animation qui diminue comme une peau de chagrin. Cette désaffectation entraîne une dégradation de la trame d'habitat en plus de provoquer une réduction de ser- vices, ce qui, en l'occurrence, contribue à accentuer la marginalisation des milieux concernés.

Si la dernière décennie s'est caractérisée par la vente de plusieurs presbytères, la prochaine pourrait bien être marquée par la fermeture d'églises. Déjà, certains temples religieux ont fermé leurs portes. C'est le cas notamment à Saint-Jean-de-Cherbourg et à SainteMarguerite, au Bas-St-Laurent, alors que d'autres sont actuellement confrontés à des choix décisifs. Le processus de transfert des responsabilités inhérent au phénomène de désaffectation des lieux de culte étant relativement nouveau, les initiatives mises en œuvre pour les préserver ne sont pas aussi courantes que celles que l'on peut observer pour d'autres types de services. En fait, dans ce contexte inédit, les questions sont beaucoup plus nombreuses que les réponses. Qu'adviendra-t-il de ces infrastructures lorsqu'elles ne seront plus utilisées à des fins religieuses ? Quels édifices devront être sacrifiés et à quel prix ? Qui prendra à charge ceux qui méritent d'être conservés ? Une nouvelle vocation est-elle possible ? Si oui, celleci est-elle compatible avec la nature du lieu, son insertion dans la trame rurale, la taille de l'édifice, son organisation spatiale, sa typologie architecturale? Changer la vocation d'une église est une entreprise à la fois délicate et complexe. En effet, la conversion d'un bâtiment patrimonial, surtout s'il est de grande taille, exige que la nouvelle vocation soit appropriée et que les transformations effectuées pour adapter l'édifice soient compatibles avec son caractère architectural et son enracinement au sein de la population.

\section{Si la dernière décennie s'est caractérisée par la vente de plusieurs presbytères, la prochaine pourrait bien être marquée par la fermeture d'églises.}

\section{Quelques exemples d'initiatives de développement local en milieu rural québécois}

Dans le diocèse de Rimouski, comme ailleurs au Québec, les responsables de l'administration des paroisses sont à la recherche de solutions concrètes et pratiques. Nombreuses sont les nouvelles vocations possibles. Dès lors, on assiste, à quelques endroits, à l'émergence d'expériences fort originales qui témoignent non seulement de la débrouillardise, de l'ardeur et de 
l'imagination qui animent le monde rural, mais aussi de la volonté de sa population à conserver son patrimoine religieux.

À notre connaissance, le cas de l'église de SainteFrançoise-Romaine, dans la région de Lobinière, constitue un précédent en ce domaine puisque l'église a été convertie en salle multifonctionnelle depuis quelques années déjà. Une partie de l'église est réservée pour le culte religieux. Dans cette même foulée, l'église paroissiale de Sainte-Ursule, dans la MRC de Maskinongé, a été transformée en logements sociaux. Toutefois, on a pris soin de conserver un espace pour les célébrations dominicales.

Dans la MRC des Basques au Bas-St-Laurent, un comité a été mis en place à Saint-Médard et à SainteRita afin de trouver une nouvelle vocation à l'église paroissiale que la compagnie d'assurance ne veut plus couvrir à moins que la fabrique ne fournisse une expertise professionnelle et effectue les travaux majeurs demandés. Des pourparlers sont présentement en cours afin que les municipalités ou un organisme communautaire puissent se porter acquéreur de ces bâtiments et leur donner une seconde vie. Le dossier n'est pas clos.

À Saint-Séverin, un petit village de la Beauce, un comité mise sur les infrastructures religieuses (église, presbytère et cimetière) afin de redynamiser la vie culturelle. Réunissant une demi-douzaine de réalisateurs, un festival du film religieux s'est récemment tenu au sein de cette municipalité. Les films ont été projetés à l'intérieur de l'église. À plus long terme, un projet est sur la table afin de la convertir en cinéma.

On retrouve aussi quelques exemples de reconversion d'églises au sein du milieu rural abitibien. À La Ferme, des modifications apportées à l'intérieur de l'église ont permis de réaliser une exposition permanente. À La Motte et à Louvicourt, l'église servira dorénavant de centre communautaire. Dans ces trois cas, la propriété de l'édifice a été cédée à la municipalité moyennant un coût symbolique. À Bellecombe, une partie de l'église a été aménagée afin d'offrir un service de garde.

En Outaouais, la petite municipalité de Luskville a accepté, devant l'insistance du marguillier en charge, de reconnaître officiellement l'église paroissiale comme un bien patrimonial qui doit être légué aux géné- rations futures, quel qu'en soit l'usage. Par cette reconnaissance, la municipalité de Luskville a le mandat de fournir la plus grande part des sommes nécessaires à la réfection du bâtiment. Une campagne de commandite a été lancée dans le but de trouver les fonds nécessaires à l'achèvement des travaux. Cette campagne, qui a soulevé un tollé, permet à des paroissiens de voir leur nom inscrit sur un élément de l'institution (fenêtre, banc, vases sacrés, etc.). Dans ce cas précis, c'est le président de la fabrique, un néo-rural, qui a joué un rôle de leader dans ce dossier. En dépit des remous qu'il a créés chez certains paroissiens de souche et des nombreuses réticences rencontrées, la communauté de Luskville était tellement fière des réalisations de son bienfaiteur qu'elle a organisé une fête en son honneur.

\section{En ce qui concerne plus particulièrement \\ les services religieux, différents scénarios sont envisagés par les populations locales en collaboration avec les curés et les agents de pastorale.}

Une autre expérience fort intéressante concerne les travaux de réfection réalisés à l'église unie Auster, située dans le canton de Clarendon, en Outaouais. Toutes les réparations, qui ont mobilisé pas moins de 25 personnes, ont été effectuées sur une base bénévole. Un seul menuisier professionnel a été engagé par la fabrique. La restauration de l'église, qui a réuni des individus de toute allégeance religieuse a, de toute évidence, été un projet mobilisateur.

Le cas de Saint-Venant-de-Paquette, une petite municipalité de 116 habitants située près de Coaticook, est également des plus novateurs puisque l'église a été rachetée par les fidèles. Des paroissiens ont menacé le diocèse de mobiliser les médias advenant la fermeture de leur temple. Malgré l'arrêt des services religieux hebdomadaires, ils ont pu conserver quelques célébrations correspondant aux temps fort de l'année liturgique (Carême, Pâques, Noël), le diocèse ayant cédé le bâtiment à un organisme sans but lucratif (Les amis du patrimoine) pour la somme d'un dollar. L'église s'autofinance notamment grâce à l'organisation de spectacles qui se tiennent depuis 1993 à raison de trois fois par année. En outre, on a aménagé un musée dans les tribunes latérales et en arrière de l'église. Un prix d'entrée est exigé. Des bingos se tiennent égale- 
ment sur une base régulière, alors qu'une collecte de fonds, dont le modèle de financement est copié sur celui du diocèse, est réalisée à tous les ans. Enfin, par souci d'économie, l'église n'est plus chauffée.

Sur la Côte-Nord, l'église de Longue-Rive abrite maintenant une entreprise de traitement de petits fruits qui emploie une douzaine d'employés. Si, dans ce cas précis, l'accès communautaire n'a pu être maintenu, cette nouvelle fonction contribue à la diversification économique d'une localité en perte de vitesse au plan démographique.

\section{La propriété collective et l'accessibilité communautaire sont les meilleurs alliés de la protection d'un patrimoine pris en charge par les communautés locales.}

Mentionnons également la conversion de l'église de Saint-Joseph-de-Ham-Sud, dans la MRC d'Asbestos, en centre d'arts. Propriété de la comédienne Rita Lafontaine, l'édifice maintient sa vocation communautaire en plus de présenter, pendant la période estivale, différents types de spectacles.

En ce qui concerne plus particulièrement les services religieux, différents scénarios sont envisagés par les populations locales en collaboration avec les curés et les agents de pastorale. L'un de ceux-ci concerne la mise en place d'un nouveau cadre territorial qui, à bien des égards, comporte moult avantages ${ }^{2}$. Il est susceptible, en effet, de promouvoir la vitalité des communautés chrétiennes et de renforcer leur cohésion sociale non seulement par le biais des célébrations dominicales, mais aussi par l'organisation d'événements festifs (les fêtes de secteur) qui réunissent les communautés de plusieurs «anciennes paroisses locales » pour n'en former qu'une seule, souvent plus dynamique et plus vivante. À l'instar des fusions municipales qui, dans bien des cas, favorisent la mise en commun de certains services essentiels, le regroupement des paroisses incite à une réorganisation des ressources pastorales à laquelle sont conviés un nombre sans cesse grandissant de laïcs.

\section{Des avenues à privilégier ?}

Dans le processus collectif de recherche, il semble que certaines orientations pourraient être privilégiées.
En autant que faire se peut, n'aurait-on pas tout avantage à prioriser le maintien d'une propriété collective pour les édifices que les paroisses ne peuvent plus soutenir seules ou dont elles doivent se départir? Le transfert d'un presbytère ou d'une église d'une fabrique vers une municipalité protège parfaitement cette dimension. Les paroissiens de Saint-Cyprien, dans la MRC de Rivière-du-Loup, viennent de conclure une entente qui fera du presbytère l'abri de la nouvelle bibliothèque municipale et, éventuellement, un lieu d'enseignement de la musique et une galerie d'exposition pour les artistes locaux. La propriété collective est assurée. L'accès communautaire est maintenu. Une nouvelle vocation éducative et culturelle a été ciblée au bénéfice du plus grand nombre. Les signataires de l'entente s'engagent à protéger le caractère patrimonial des édifices, église et presbytère, de même que l'espace vert qui les entourent, par la création éventuelle d'un parc municipal. Un projet similaire est sur la table dans la paroisse voisine de Saint-Clément. En milieu rural, de telles transactions entre deux corporations comme une fabrique et une municipalité permettent de conclure des ententes où un soutien peut être accordé à la fabrique en vue du maintien de l'église au titre d'édifice à vocation communautaire. Ce type de transaction gagnerait à être étendu aux églises qui sont le fruit du labeur et de la générosité collective. La privatisation complète ou encore la démolition devraient être les dernières options, surtout en milieu rural. En ce domaine, les différentes formes de partenariat nous apparaissent une avenue incontournable à privilégier, car elles représentent une condition essentielle dans la prestation des services de proximité en milieu rural ${ }^{3}$.

La propriété collective et l'accessibilité communautaire sont les meilleurs alliés de la protection d'un patrimoine pris en charge par les communautés locales. Par ailleurs, dans les aménagements encore à inventer ou à créer, le développement de solutions de financement à même l'utilisation d'une certaine partie des lieux à préserver peut être envisagé dans plusieurs cas. Outre l'utilisation de l'espace par des organismes communautaires qui justifierait alors l'intervention des collectivités locales, régionales ou même de l'État, peut-on envisager la location d'espaces qui serviraient à apporter un soutien financier au bâtiment que l'on cherche à conserver ? À ce titre, aucune église ne possède la même physionomie ou les mêmes possibilités; toutefois, plusieurs bénéficient de soussols facilement aménageables pour répondre aux be- 
soins d'une société de comptables ou d'assureurs, d'une garderie, d'une résidence funéraire ou encore d'un cabinet de professionnels. Dans plusieurs cas, c'est même une partie importante de la nef qui pourrait être convertie, tout en préservant le chœur et les transepts qui pourraient ainsi abriter un espace sacré réservé au culte. La créativité déjà à l'œuvre depuis plusieurs décennies nous invite à penser qu'il y a autant de solutions possibles que de situations qui constituent des cas d'espèce.

\section{Conclusion}

La fermeture d'église dans un petit village n'a pas le même impact que dans un centre urbain. Il n'est pas question ici de rouvrir le débat sur ce qui différencie le rural et l'urbain au plan sociologique ou géographique, mais simplement de signifier le choix qui se présente aux Québécois et de mesurer les conséquences d'une privatisation laissée au libre marché et aux seules considérations économiques. Les enjeux sont à la fois historiques, patrimoniaux, sociaux et territoriaux. Il y a fort à parier que l'avenir de certains joyaux dispersés dans le monde rural sera compromis faute de population et de ressources suffisantes. Que serait le paysage architectural de la France si elle n'avait sauvegardé que les châteaux situés au centre ou en périphérie des grands centres comme Paris ou Lyon?

Les symboles, même s'ils ne nous relient pas tous aux mêmes réalités, sont toujours pertinents dans le monde moderne, et chaque région peut prétendre à préserver les siens. Collectivement, nous pourrions être privés d'un riche héritage si l'on bradait trop facilement le patrimoine religieux de l'Église catholique au
Québec. L'heure est peut-être à une nouvelle répartition des charges. L'Église et la cité, sur ce point précis, doivent trouver un point de rencontre. Pour y arriver, les débats francs et ouverts sont nécessaires, de même que l'établissement de nouveaux partenariats entre les différents acteurs du milieu local, régional et national. La sauvegarde du patrimoine religieux représente un enjeu fondamental pour le monde rural québécois. Les initiatives que nous avons citées dans le cadre de cet article doivent servir de prémisses dans la mise en œuvre de nouvelles stratégies d'action. De toute évidence, une nouvelle gouvernance territoriale est en formation en milieu rural.

\section{Notes et références}

1 À Saint-Nil, une municipalité du Bas-St-Laurent fermée dans les années 1970 à la suite des recommandations du Bureau d'aménagement de l'Est-du-Québec (BAEQ), le cimetière est tellement chargé de significations et de symboles que des anciens résidents veillent, chaque été, à ce qu'il soit bien aménagé et entretenu.

2 Concernant ce nouveau découpage, on lira avec intérêt les deux articles suivants de Claude Pigeon : "Genèse d'un secteur pastoral. Le nouveau visage de cinq paroisses rurales dans le Bas-Saint-Laurent », dans L'Action nationale, vol. $94, n^{\circ} 9$ et 10 (novembre-décembre 2004) p. 152-174 et "Une semaine sainte en secteur pastorale », paru dans Liturgie, foi et culture, vol. 39, $\mathrm{n}^{\circ} 183$ (automne 2005), p. 17-20.

3 À ce titre, on pourra consulter l'étude suivante : Simard, M. (2005), Les services de proximité en milieu rural québécois : synthèse des connaissances, Rimouski, Chaire de recherche du Canada en développement rural, octobre, $48 \mathrm{p}$. 